\title{
Ambiguity Analysis and Resolution for Phase-Based 3D Source Localization under Given UCA
}

\author{
Zhen Liu, ${ }^{1}$ Xin Chen ${ }^{(D},{ }^{1}$ Zhenhua Wei, ${ }^{2}$ Tianpeng Liu, ${ }^{1}$ Linlin Li, ${ }^{2}$ and Bo Peng ${ }^{1}$ \\ ${ }^{1}$ College of Electronic Science, National University of Defense Technology, Changsha 410073, China \\ ${ }^{2}$ Rocket Force University of Engineering, Xian 710025, China \\ Correspondence should be addressed to Xin Chen; chenxin10@nudt.edu.cn
}

Received 31 August 2018; Revised 21 March 2019; Accepted 7 April 2019; Published 18 April 2019

Academic Editor: Hervé Aubert

Copyright (c) 2019 Zhen Liu et al. This is an open access article distributed under the Creative Commons Attribution License, which permits unrestricted use, distribution, and reproduction in any medium, provided the original work is properly cited.

Under uniform circular array, by employing some algebraic schemes to exploit the phase information of receiving data and further estimate the source's three-dimensional (3D) parameters (azimuth angle, elevation angle, and range), a series of novel phase-based algorithms with low computational complexity have been proposed recently. However, when the array diameter is larger than source's half-wavelength, these algorithms would suffer from phase ambiguity problem. Even so, there always exist certain positions, where the source's parameters can still be determined with nonambiguity. Therefore, this paper first investigates the zone of ambiguity-free source 3D localization using phase-based algorithms. For the ambiguous zone, a novel ambiguity resolution algorithm named ambiguity traversing and cosine matching (ATCM) is presented. In ATCM, the phase differences of centrosymmetric sensors under different ambiguities are utilized to match a cosine function with sensor number-varying, and the source's unambiguous rough angles can be derived from amplitude and initial phase of the cosine function. Then, the unambiguous angles are employed to resolve the phase ambiguity of the phase-based 3D parameter estimation algorithm, and the source's range as well as more precise angles can be achieved. Theoretical analyses and numerical examples show that, apart from array diameter and source's frequency, the sensor number and spacing of employed sensors are two key factors determining the unambiguous zone. Moreover, simulation results demonstrate the effectiveness and satisfactory performance of our proposed ambiguity resolution algorithm.

\section{Introduction}

Passive source localization using an array of sensors has numerous key applications for wireless communication, electron reconnaissance, astronomy, smart antennas, etc. [1-5]. A uniform circular array (UCA) has advantages over the other array geometries due to its $360^{\circ}$ azimuth coverage, an almost identical beamwidth, and additional elevation angle information [6-14].

In the context of three-dimensional (3D) parameter estimation, by employing some algebraic schemes to exploit the phase information of receiving data and further estimate the source's 3D parameters, a series of novel phase-based algorithms using UCA have been proposed in [11-14], which are computationally simple and also provide acceptable estimation performance. Comparing with traditional schemes such as the Multiple Signal Classification (MUSIC) and Estimation of Signal Parameters via Rotational Invariance Techniques (ESPRIT) algorithms, the phase-based algorithms have obvious advantage on the computational complexity. However, when the array diameter of UCA exceeds the half-wavelength of source, periodical ambiguities would be introduced into phase difference measurement of these algorithms. Even so, it should be noticed that there still exists some accurate estimation of source's location under ambiguous situations and the correct estimation zone probably accords with the demand of practical application. Therefore, under ambiguous situations, it is significant to analyze the zone of ambiguity-free before using the phase-based algorithms. As for ambiguity-free zone, Tan et al. [15] presented the ambiguity in the MUSIC and ESPRIT algorithms for 2D direction of arrival estimation under a uniform linear array and provided the ambiguity-free zones of these two algorithms. So far, aiming to the phasebased algorithms, ambiguous analysis has not been found in 


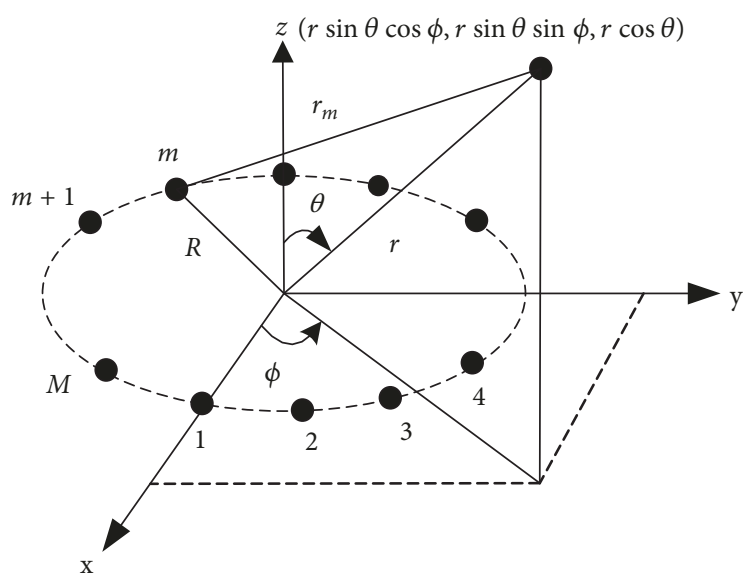

FIGURE 1: Geometry of circular array and source.

existing literatures. Moreover, when the source is located at ambiguous zone, effective methods of ambiguity resolution are necessary to obtain accurate source's location. In the case of ambiguity resolution for the phase-based algorithm under UCA, Xin et al. [16] proposed two different algorithms for unambiguous localization of a single far-field source based on two user-defined types of phase difference observation models. This two methods can achieve unambiguous DOA estimation. However, they only consider the localization of a single far-field source and near-filed situation is inapplicable to the proposed methods. As for the near-filed situation of unambiguous source's 3D localization, we recently proposed a feasible scheme. In [17], by referring to the idea of ambiguity resolution via rotating interferometer, we employ adaptive rotation to make the sensors form virtual short baseline to resolve phase ambiguity of stationary monofrequency sources. However, moving or frequency-hopping sources are common in various environment, this rotary way is inapplicable as the matching of source's frequency and location before and after rotation are demanded and relevant methods need to be presented.

Accordingly, in this paper, we first analyze the ambiguity problem of phase-based algorithms, and the correct estimate zone of source's 3D localization under ambiguous situations is then investigated, which could provide useful guidance in practical appliance. Thereafter, we present a novel algorithm of ambiguity resolution named ambiguity traversing and cosine matching (ATCM), which can achieve both monofrequency and frequency-hopping sources' unambiguous localization. Herein, source's angles and range are first decoupled by using the centrosymmetry of UCA with even number of sensors. Then, by ambiguity traversing, a matrix of phase difference under different ambiguities is developed. After that, based on the cosine property of unambiguous phase differences, all elements of the phase difference matrix are utilized to match with a cosine function with variable amplitude and initial phase, which correspond to source's angles. By employing the method of exhaustive searching, the optimal matching can be found and its corresponding rough angle estimation with no ambiguity is obtained subsequently. Finally, to realize source's range as well as more precise angle estimation, we proposed an effective method in [18] by utilizing the obtained unambiguous angles to resolve ambiguity in the phase-based algorithm via the approximation of steering vectors between planar wavefront and curved wavefront, which can be also applied here.

This paper contributes to the area of 3D source localization in the following three aspects:

(1) We analyze the ambiguity problem of the phase-based algorithms and present the ambiguity-free estimation zone under ambiguous situations which could provide guidance in practical appliance.

(2) For source's 3D localization, this paper provides a novel ambiguity resolution method to obtain actual source's parameters with satisfactory performance and acceptable computational complexity.

(3) As the proposed algorithm does not need rotation, it can achieve real-time source's localization by only once implementation, and the applied scope is wider than rotary way to resolve ambiguity.

The rest of the paper consists of six sections. Section 2 reviews the model of a single narrowband source's 3D parameter estimation. Section 3 presents the ambiguity problem of phase-based algorithms and gives the equation of ambiguity-free estimation zone under ambiguous situations. Section 4 introduces the method of ATCM to obtain source's unambiguous angle estimation. Section 5 carries out simulations to demonstrate the rationality of theoretic analysis on unambiguous estimation zone and the effectiveness of the proposed ATCM algorithm. Section 6 concludes the whole paper.

\section{Signal Modeling}

As shown in Figure 1, a fixed UCA with radius $R$ and $M$ identical omnidirectional sensors is employed, where the $M$ sensors are uniformly spaced on the circumference in the 
$x y$-plane. Assuming that a single narrowband source with curved wavefront is located at $(\phi, \theta, r)$, where the azimuth angle $\phi \in[-\pi, \pi]$ is measured counterclockwise from the $x$ axis, the elevation angle $\theta \in[0, \pi / 2]$ is measured downward from the $z$-axis, and the range $r$ is measured from the center of the UCA.

Without loss of generality, we consider the source's range is beyond the Fresnel area but dissatisfies the condition of farfield. Under these assumptions, the $m$ th output of the sensor at time $n$ can be written as

$$
x_{m}(n)=s(n) \exp \left\{j \frac{2 \pi}{\lambda}\left(r-r_{m}(\phi, \theta, r)\right)\right\}+w_{m}(n)
$$

for $m=1, \ldots, M, n=1, \ldots, N . s(n)$ is the complex envelope with power $\sigma_{s}^{2}$, and $w_{m}(n)$ is the white complex Gaussian noise vector with power $\sigma_{n}^{2}$, which is independent of the $s(n)$. $\lambda$ is the wavelength of the source. $r_{m}(\phi, \theta, r)$ is the range between the $m$ th sensor and the source, which has the form of

$$
r_{m}(\phi, \theta, r)=r \sqrt{1+\left(\frac{R}{r}\right)^{2}-\frac{2 R \zeta_{m}(\phi, \theta)}{r}}
$$

where $\zeta_{m}(\phi, \theta)=\cos \left(\gamma_{m}-\phi\right) \sin \theta$ with $\gamma_{m}=2 \pi(m-1) / M$. Consider a function defined as

$$
f\left(\iota_{r}\right)=\left\{1+\iota_{r}^{2}-2 \iota_{r} \cdot \zeta_{m}(\phi, \theta)\right\}^{1 / 2}
$$

where $t_{r}=R / r$. According to a sufficiently large $r$ relative to $R, f\left(t_{r}\right)$ can be well approximated by a second-order Taylor series expansion as

$$
f\left(\iota_{r}\right) \approx f(0)+f^{\prime}(0) \iota_{r}+\frac{1}{2} f^{\prime \prime}(0) \iota_{r}^{2}
$$

Then, $r_{m}(\phi, \theta, r)$ can be approximated as

$$
r_{m}(\phi, \theta, r) \approx r-R \zeta_{m}(\phi, \theta)+\frac{R^{2}}{2 r}\left(1-\zeta_{m}^{2}(\phi, \theta)\right)
$$

Substituting (5) into (1) yields the approximated signal model as

$$
\begin{aligned}
& x_{m}(n) \\
& =s(n) \exp \left\{j \frac{2 \pi R}{\lambda}\left(\zeta_{m}(\phi, \theta)-\frac{R}{2 r}\left(1-\zeta_{m}^{2}(\phi, \theta)\right)\right)\right\} \\
& \quad+w_{m}(n)
\end{aligned}
$$

It can be seen from (6) that the source's angles and range are included in its exponent. By employing some algebraic schemes to exploit the phase information of receiving data and further estimate the parameters of angles as well as range, source's 3D localization can be well achieved and we define this kind of technology as the phase-based algorithms.

\section{Ambiguity Analysis of Phase-Based Algorithms}

Recently, for source's 3D localization, phase-based algorithms receive growing attention as its low computational complexity and high estimation precision. However, when the array diameter exceeds half of source's wavelength, phase-based algorithms would suffer from ambiguity problem. It should be pointed out that not all source's location estimation would appear phase ambiguity. Here, we first introduce the phasebased algorithms. After that, we analyze the ambiguity problem of phase-based algorithms and the still correct estimation zone under ambiguous situations is then investigated.

3.1. Introducing of the Phase-Based Algorithms. In phasebased algorithms, a correlation function is always defined as

$$
\begin{aligned}
R_{m, d} & =E\left\{x_{m}(n) x_{d}(n)^{*}\right\} \\
& =\sigma_{s}^{2} \exp \left\{\mathrm{j} \frac{2 \pi R}{\lambda}\left(\psi_{m}(\phi, \theta, r)-\psi_{d}(\phi, \theta, r)\right)\right\}+\sigma_{n}^{2}
\end{aligned}
$$

where $E\{\}$ denotes the expected value,

$$
\begin{aligned}
& d \\
& = \begin{cases}M, & \text { for } m+l=M, l=1,2, \ldots, M-1 \\
\bmod (m+l, M), & \text { otherwise }\end{cases}
\end{aligned}
$$

$l$ represents the spacing between employed sensors, $\psi_{m}(\phi$, $\theta, r)=\zeta_{m}(\phi, \theta)-(R / 2 r)\left(1-\zeta_{m}^{2}(\phi, \theta)\right)$, and $(\cdot)^{*}$ denotes the complex conjugate. Assuming that the receiving data is noiseless, the phase of $R_{m, d}$ is

$$
u_{m, d}=\frac{2 \pi R}{\lambda}\left(\psi_{m}(\phi, \theta, r)-\psi_{d}(\phi, \theta, r)\right)+2 \pi q
$$

where $q$ is a certain integer. Thereafter, under unambiguous situation, the phase-based algorithms reformulate unambiguous phase angle as the form of matrix and utilize the least square (LS) schemes to obtain source's 3D parameters [11-13].

3.2. Ambiguity Problem of Phase-Based Algorithms. It should be pointed out that the exploited phase of correlation function belongs to $[-\pi, \pi)$ and the value is inconsistent to the real phase when $q \neq 0$, which would cause incorrect estimation of source's location parameters. Therefore, the situation that ineffectiveness of source's localization caused by incorrect phase estimation as $q \neq 0$ is called phase ambiguity. To guarantee no phase ambiguity in (9), the condition $R \leq \lambda / 4$ is always necessary to ensure $q=0$ since $\mid \psi_{m}(\phi, \theta, r)-$ $\psi_{d}(\phi, \theta, r) \mid \leq 2$.

It is worth noting that once the array has been designed, the limitation $R \leq \lambda / 4$ is useless for localization of overfrequency source. However, not all the estimated positions of over-frequency sources would appear phase-ambiguous and it is significant for practical demand to investigate the correct estimation zone of source's locations under ambiguous situations.

3.3. Unambiguous Estimation Zone of Source's Locations. Under ambiguity-free situation $q=0$,

$$
\rho_{m, d}=\frac{2 \pi R}{\lambda}\left(\psi_{m}(\phi, \theta, r)-\psi_{d}(\phi, \theta, r)\right)
$$


By employing trigonometric function, the unambiguous phase difference $\rho_{m, d}$ can be easily simplified as

$$
\begin{aligned}
& \psi_{m}(\phi, \theta, r)-\psi_{d}(\phi, \theta, r)=\zeta_{m}(\phi, \theta)-\zeta_{d}(\phi, \theta) \\
&+ \frac{R}{2 r}\left(\zeta_{d}^{2}(\phi, \theta)-\zeta_{m}^{2}(\phi, \theta)\right)=\sin \theta\left(\cos \left(\gamma_{m}-\phi\right)\right. \\
&-\left.\cos \left(\gamma_{d}-\phi\right)\right)+\frac{R}{2 r} \sin ^{2} \theta\left(\cos ^{2}\left(\gamma_{d}-\phi\right)\right. \\
&-\left.\cos ^{2}\left(\gamma_{d}-\phi\right)\right) \\
& \rho_{m, d}=\frac{4 \pi R}{\lambda}\{\sin (\chi(m+d-2)-\phi) \sin (\chi(d-m)) \\
& \cdot \sin \theta+\frac{R}{2 r} \cdot \sin (2 \chi(d-m)) \\
&\left.\cdot \sin (2 \chi(m+d-2)-2 \phi) \sin ^{2} \theta\right\}
\end{aligned}
$$

where $\chi=\pi / M$. According to a sufficiently large value of $r$ relative to $R,(12)$ can be well approximated as

$$
\begin{aligned}
& \rho_{m, d} \\
& \quad \approx \frac{4 \pi R}{\lambda} \sin (\chi(m+d-2)-\phi) \sin (\chi(d-m)) \sin \theta
\end{aligned}
$$

As the phase difference $u_{m, d}^{\prime}$ is unambiguous, it satisfies $\left|u_{m, d}^{\prime}\right| \leq \pi$. Substituting (13) into the condition yields

$$
\begin{aligned}
& \sin (\theta) \\
& \leq \frac{\lambda}{\{4 R \cdot|\sin (\chi(d-m)) \sin (\chi(m+d-2)-\phi)|\}}
\end{aligned}
$$

To further simplify the expression above, we take no account of the influence of source's azimuth angle by setting $\sin (\chi(m+d-2)-\phi)=1$, and (14) can be expressed as

$$
\sin (\theta) \leq \frac{\lambda}{\{4 R \cdot|\sin (\chi(d-m))|\}}
$$

And then,

$$
\theta_{\max } \approx \arcsin \left\{\frac{\lambda}{\left\{4 R \cdot \max _{m}(|\sin (\chi(d-m))|)\right\}}\right\}
$$

It can be found that the maximum elevation angle of source is related to the number of sensors, the array diameter, the choice of spacing $l$, and the source's frequency. Therefore, under the maximum elevation angle of source determined by certain factors in (16), the phase differences in phase-based algorithms would not introduce ambiguity and the parameter estimation of related source's locations is effective. However, the ambiguous zone is wide and methods of resolving ambiguity need to be proposed. Thus, in the next section, we would present a novel ambiguity resolution algorithm based on the cosine property of phase differences, whose part work has been published in [19].

\section{Ambiguity Resolution by Using the Method of ATCM}

It is clear that incorrect range estimation is caused by large angle estimation errors, so ambiguity in 3D parameter estimation of source is mainly brought from angle estimation. However, looking at (6), ambiguity resolution is an intractable task as source's angles interweave with the range. Therefore, the troublesome separation of source's angles and range is demanded to realize ambiguity resolution. Here, we first define two sensors which are symmetric about the centre of a circle as the centrosymmetric sensors (CCSs), so the number of sensors must be even to ensure this property. And source's angles and range are decoupled by computing the phase differences of CCSs. Then, based on the cosine property of unambiguous phase differences, the method of ambiguity traversing and actual-value matching are employed to obtain rough source's angle estimation with no ambiguity.

4.1. Separation of Source's Angles and Range. It can be noticed that $\gamma_{m+M / 2}=\gamma_{m}+\pi$ under a UCA with even sensors, and then

$$
\zeta_{m+M / 2}(\phi, \theta)=-\zeta_{m}(\phi, \theta)
$$

for $m=1,2, \ldots, M / 2$. According to (9), the phase differences of CSSs can be expressed as

$$
\begin{aligned}
u_{m, m+M / 2}= & \frac{2 \pi R}{\lambda}\left(\psi_{m}(\phi, \theta, r)-\psi_{m+M / 2}(\phi, \theta, r)\right) \\
& +2 \pi q=\frac{4 \pi R \zeta_{m}(\phi, \theta)}{\lambda}+2 \pi q
\end{aligned}
$$

Note that $u_{m, m+M / 2}$ is only represented by the $2 \mathrm{D}$ angle parameter $\zeta_{m}(\phi, \theta)$ of the source's locations and effective methods of resolving ambiguity can be utilized to obtain actual source's angles under ambiguous situation with $q \neq 0$.

4.2. Ambiguity Resolution of Source's Angles. The unambiguous phase differences $\rho_{m, m+M / 2}=4 \pi R \sin \theta \cos (2 \pi(m-$ $1) / M-\phi) / \lambda$ can be regarded as some sampling points of a cosine function, where the angular frequency is $2 \pi / M$, initial phase is $-\phi$, and the amplitude is $4 \pi R \sin \theta / \lambda$. Therefore, we can develop an optimal equation, which is given by

$$
\begin{aligned}
\min _{A, \varphi} & \sum_{m=1}^{M / 2}\left|\rho_{m, m+M / 2}-A \cos \left(\frac{2 \pi(m-1)}{M}+\varphi\right)\right| \\
\text { s.t. } & A>0, \quad \varphi \in[-\pi, \pi)
\end{aligned}
$$

where $\min \{\cdot\}$ denotes the minimum value. It is obvious that the actual source's angles can be obtained by searching the minimum in (19).

Before searching the optimal matching, the probable target value $\rho_{m, m+M / 2}$ should be firstly determined, so the maximum ambiguity of phase differences is computed as

$$
D=\operatorname{ceil}\left(\frac{2 R}{\lambda}\right)
$$


where ceil(.) denotes upper nearest integer. By ambiguity traversing, all probable unambiguous phase differences are included in the following matrix, which is given by

$$
\mathbf{U}=\left[\begin{array}{cccc}
u_{1,1+M / 2}-2 \pi D & u_{2,2+M / 2}-2 \pi D & \cdots & u_{M / 2, M}-2 \pi D \\
u_{1,1+M / 2}-2 \pi(D-1) & u_{2,2+M / 2}-2 \pi(D-1) & \cdots & u_{M / 2, M}-2 \pi(D-1) \\
\vdots & \vdots & \cdots & \vdots \\
u_{1,1+M / 2}+2 \pi D & u_{2,2+M / 2}+2 \pi D & \cdots & u_{M / 2, M}+2 \pi D
\end{array}\right]
$$

Due to uncertain amplitude and initial phase of cosine function, the method of exhaustive searching cosine matching is employed to obtain the phase differences with no ambiguity. Assuming that the beginning amplitude is $A_{0}=0$, the search step is $\Delta A$ and the upper bound is ceil $(4 \pi R / \lambda)$. Meanwhile, the beginning phase is $\varphi_{0}=-\pi$, the search step is $\Delta \varphi$, and the upper bound is $\pi$. To ascertain the unambiguous phase differences, a new optimal equation is developed, which is given by

$$
\begin{aligned}
G & (A, \varphi) \\
& =\sum_{m=1}^{M / 2} \min _{A, \varphi}\left\{\left|\mathbf{U}(\cdot, m)-A \cos \left(\frac{(2 m-1) \pi}{M}+\varphi\right)\right|\right\}
\end{aligned}
$$

where $\mathbf{U}(\bullet, m)$ denotes the elements of the $m$ th column in (21). By searching the minimum value of $G(A, \varphi)$, we can find the unambiguous phase differences, and the actual source's azimuth angle and elevation angle can be obtained by their corresponding amplitude and initial phase, which can be expressed as

$$
\begin{aligned}
& \widehat{\phi}=-\varphi \\
& \widehat{\theta}=\arcsin \left(\frac{A \lambda}{4 \pi R}\right)
\end{aligned}
$$

The procedure of ATCM could be summarized as

Step 1. By computing the phase differences of CCSs, source's angles and range can be decoupled.

Step 2. To ascertain the scope of ambiguity, the maximum ambiguity $D$ of phase differences needs to be calculated according to (18).

Step 3. After obtaining the matrix of phase differences, we develop the optimal equation and find its minimum by using the method of exhaustive searching and cosine matching.

Step 4. The ambiguity-free estimation of source's angles $(\widehat{\phi}, \widehat{\theta})$ is obtained by substituting the certain phase and amplitude into (21) and (22).

It should be pointed out that the effectiveness of resolving ambiguity and estimation precision depend on the step size in search. The smaller the step, the more accurate the estimation. However, by employing small step, the computational cost is too huge to implement real-time source's localization. In [18], we have proposed an effective solution by utilizing the obtained rough source's angles to resolve ambiguity in the phase-based algorithm via the approximation of steering vectors between planar wavefront and curved wavefront, which can be also applied here. Therefore, the ATCM algorithm first employs proper step size in search to obtain rough source's angles, and more precise angle as well as range estimation could be realized by employing our proposed way in [18], which could alleviate the conflict brought by computational complexity and estimation performance. Moreover, the aforementioned method aims to single source. As for multiple incoherent sources, we have also proposed a feasible scheme in [17] by computing the phase differences of receiving data's corresponding spectrum peak. Thereafter, by using the ATCM algorithm, each source's unambiguous 3D parameters could be estimated. The flow chart of source's unambiguous 3D localization is shown in Figure 2.

\section{Numerical Examples}

In this section, we report on simulation experiments that have been performed to demonstrate the rationality of theoretical analysis on unambiguous estimation zone and the effectiveness of the proposed method for resolving ambiguity.

Simulation experiments are divided into four parts. In Section 5.1, the comparison of theoretical analysis on unambiguous estimation zones and simulative results under threedimension situation is presented. Then, to further demonstrate the rationality of (15), the similar comparison under two-dimension situation is illustrated. In Section 5.2, we mainly validate the effectiveness of the resolving ambiguity method ATCM. Considering the influence of step size in search and signal-to-noise ratio (SNR), the performance of ATCM algorithm is evaluated in Section 5.3. Finally, in Section 5.4, we employ the histogram distribution to demonstrate the satisfactory precision of source's $3 \mathrm{D}$ parameter estimation.

5.1. Comparison of Unambiguous Parameter Estimation Zone under Three-Dimensional Situation. Without loss of generality, we consider a single source with frequency $f=900 \mathrm{MHz}$, the fixed radius of UCA $R=0.5 \mathrm{~m}$, sampling rate $f_{s}=2 \mathrm{GHz}$, and snapshots $N=2000$. It should be pointed out that the array diameter is larger than the source's half-wavelength and the parameter estimation of phase-based algorithms probably 


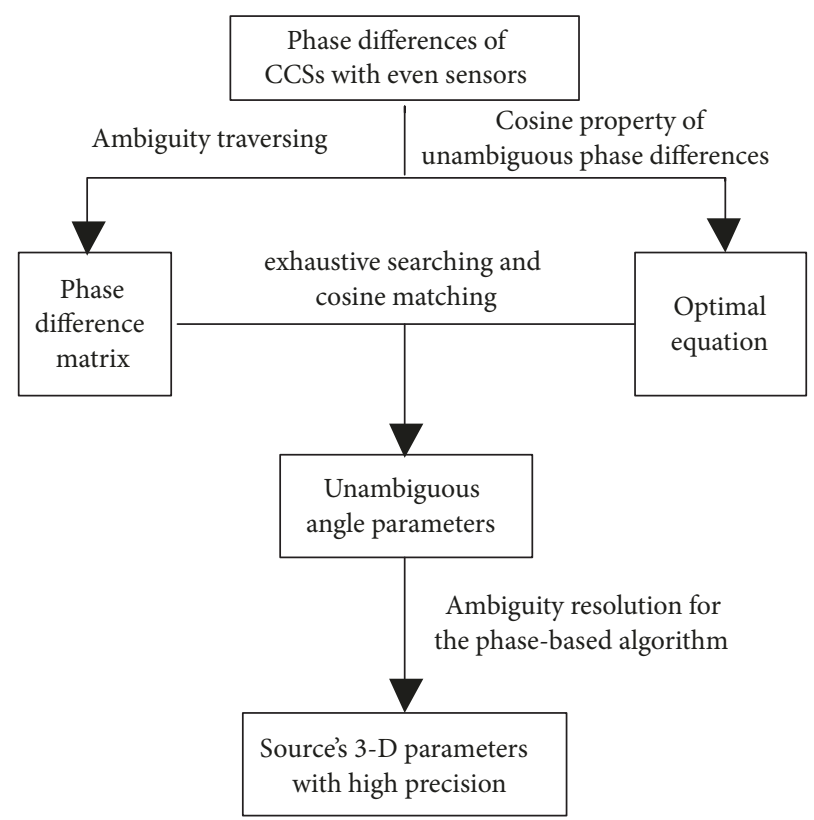

FIgURE 2: Flowchart of source's unambiguous 3D localization.

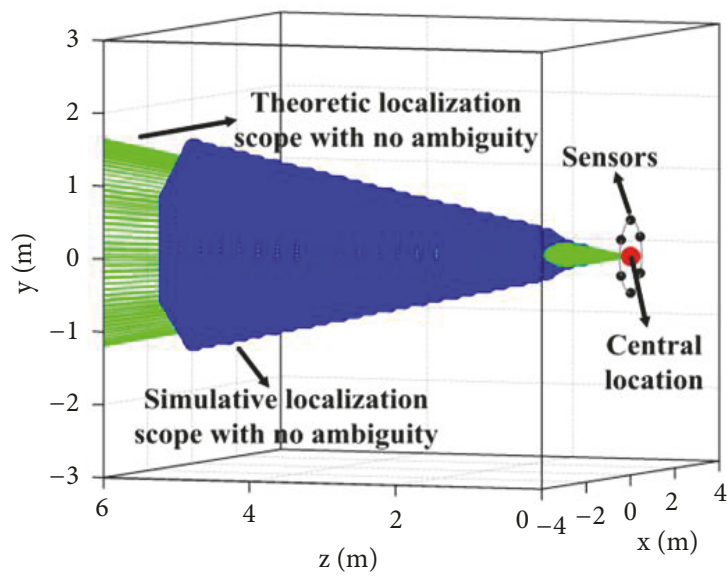

(a)

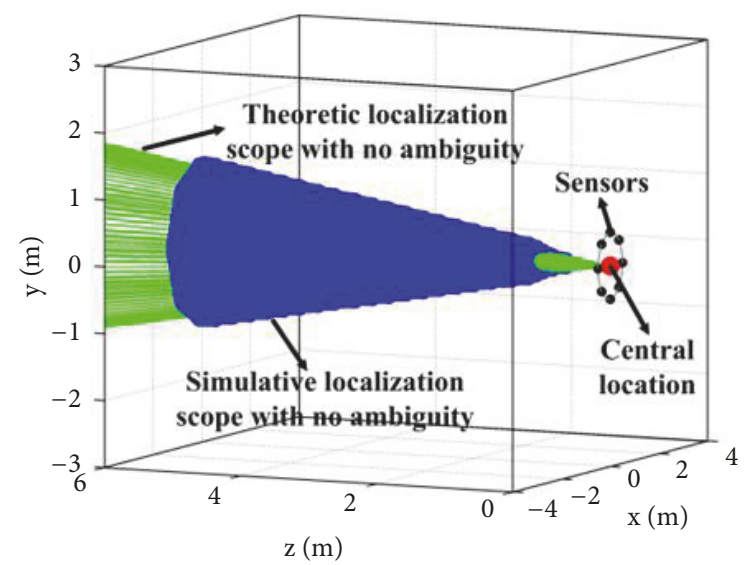

(b)

FIGURE 3: Comparison of unambiguous parameter estimation zones under three-dimensional situation. (a) $M=6, l=2$. (b) $M=8, l=3$.

introduces phase ambiguity. To verify the validity of derived analytical expression, we consider two different combinations of sensors' number and spacing, respectively.

The comparison of unambiguous parameter estimation zones computed by analytical expression and performed by simulation experiments under noise-free circumstance are shown in Figure 3. Note that, in Figure 3(a), six sensors displayed as black dots are uniformly spaced on the UCA and the red dot represents the central location of the UCA. We assume that the searching region of source location is $x \in[-4,4] \mathrm{m}, y \in[-3,3] \mathrm{m}$, and $z \in[0,6] \mathrm{m}$ and employ phase differences of sensors whose spacing is $l=2$ to obtain source's $3 \mathrm{D}$ parameter estimation. The zone covered by green lines is computed by the formula (16) and the zone covered by blue dots representing the correct estimation of current location based on the phase-based algorithm is performed by simulation experiments. It can be noticed that these two zones are almost overlapped and the simulation results accord with analytical expression. It should be pointed out that there exists a threshold of source's range for accurate $3 \mathrm{D}$ localization since a sufficiently large value of source's range is relative to array radius to ensure the feasible approximation in (5) and (13). In Figure 3(b), the number of sensors is $M=8$, the employed space between sensors is $l=3$, and other typical scenario settings keep unchanged. It can be found that the presented phenomena are similar to Figure 3(a). Accordingly, 


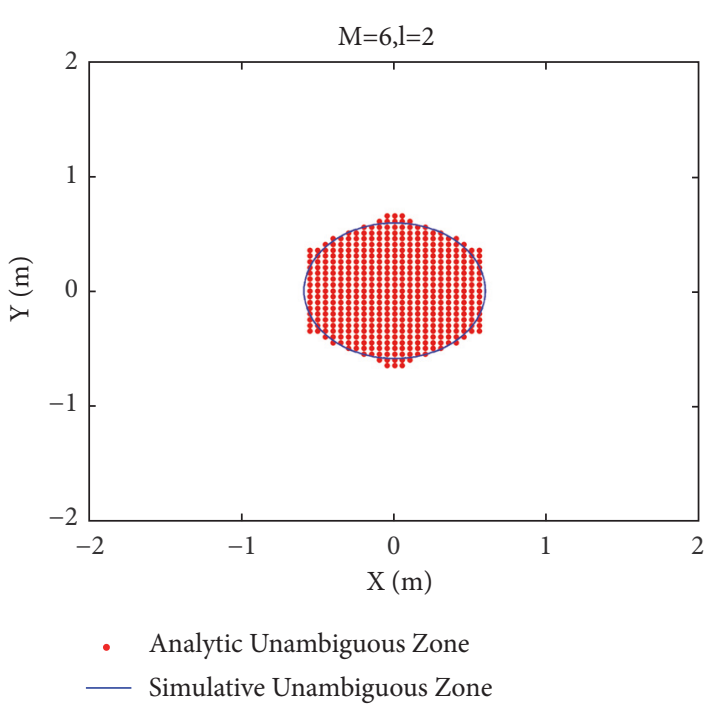

(a)

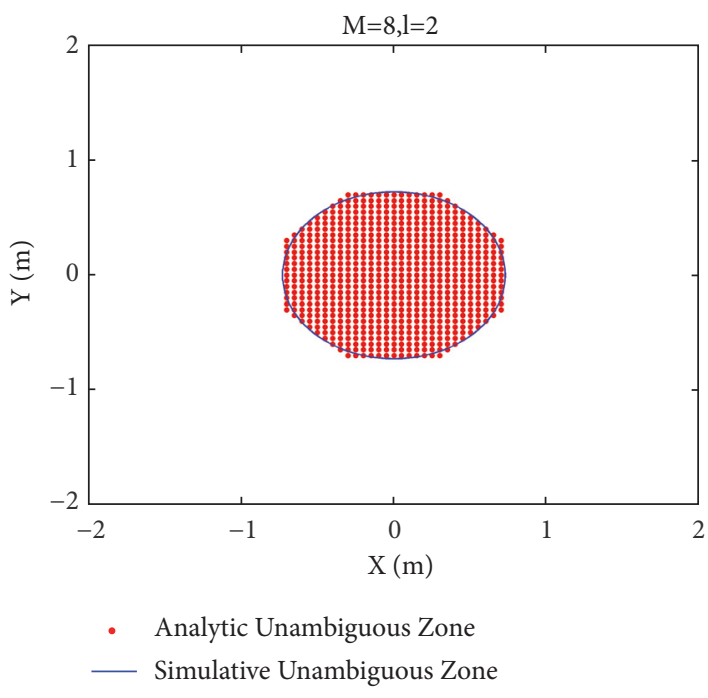

(c)

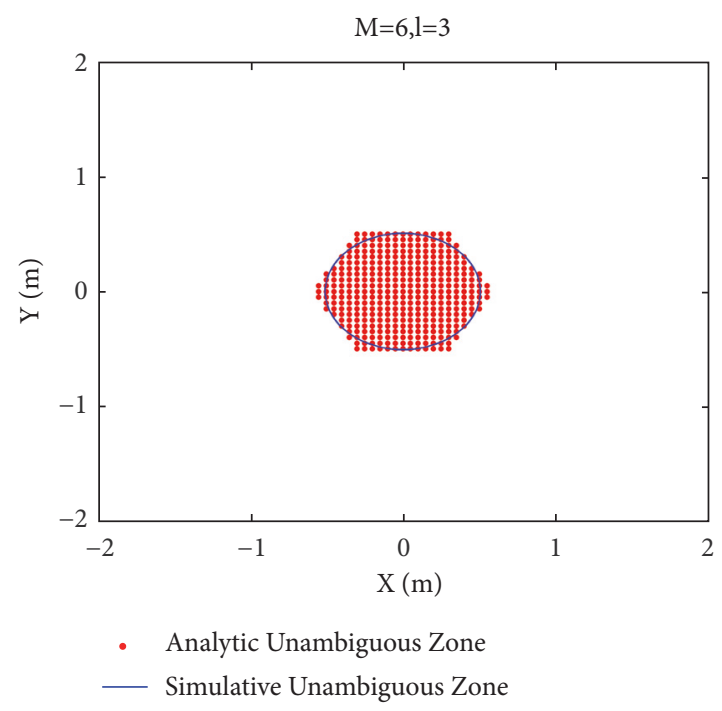

(b)

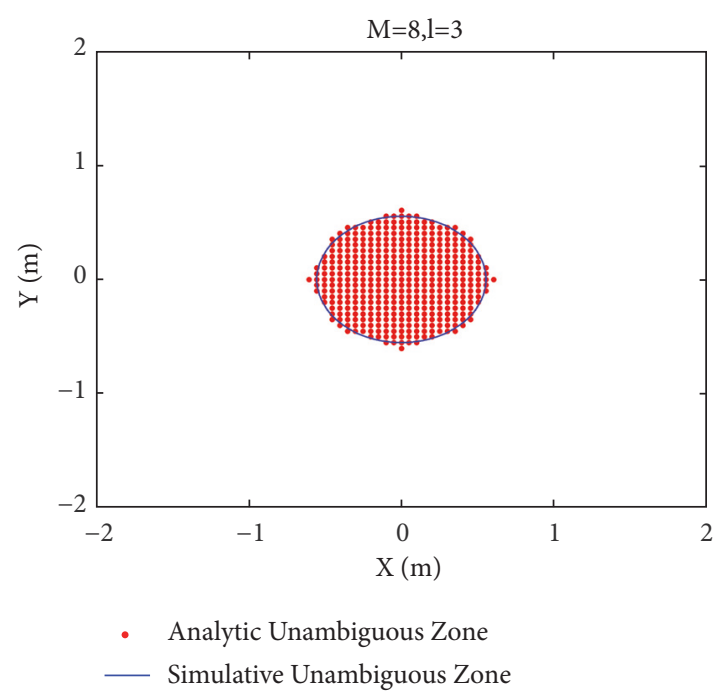

(d)

FIgURE 4: Comparison of unambiguous parameter estimation zones in the Z-plane. (a) Unambiguous zone under $M=6, l=2$. (b) Unambiguous zone under $M=6, l=3$. (c) Unambiguous zone under $M=8, l=2$. (d) Unambiguous zone under $M=8, l=3$.

we can reach the conclusion that the analytical expression can provide useful guidance to determine the unambiguous estimation zone.

Actually, we can notice that unambiguous localization zones obtained by analytical expression and simulation experiments in Figure 3 are not entirely overlapped. Therefore, we further investigate the ambiguity-free estimation zones in the Z-plane by employing $z=3 \mathrm{~m}$. As shown in Figure 4, the unambiguous estimation zones obtained by analytical expression are encircled by blue circles, and the red dots representing the estimation locations with nonambiguity are performed by simulation experiments. It can be seen that, under fixed array diameter and source's frequency, the ambiguity-free estimation zone is wider with increasing sensors but is narrower against the longer spacing of employed sensors. Further, it can be found that some unambiguous estimation positions obtained by simulations oversteps the area of analytical expression, which are caused by the approximation from (14) to (15), where the trivial influence of source's azimuth angle is neglected.

5.2. The Effectiveness of ATCM. We take two incoherent sources with identical amplitude as an example. The number of sensors is set as $M=8, \mathrm{SNR}$ is $10 \mathrm{~dB}$, the sampling rate is $f_{s}=4 \mathrm{GHz}$, snapshots $N=4000$, and other experimental parameters keep unchanged. A source with $f=900 \mathrm{MHz}$ is located at $\left(20.1^{\circ}, 10.5^{\circ}, 4.5 \mathrm{~m}\right)$ and the other one with $f=$ $1.5 \mathrm{GHz}$ is located at $\left(120.5^{\circ}, 30.2^{\circ}, 8 \mathrm{~m}\right)$. As shown in Figure 5, the blue dot is the source and the zone covered by green lines is also computed by the formula (16), where the spacing $l=2$. It is obvious that the location of source with $f=900 \mathrm{MHz}$ is 


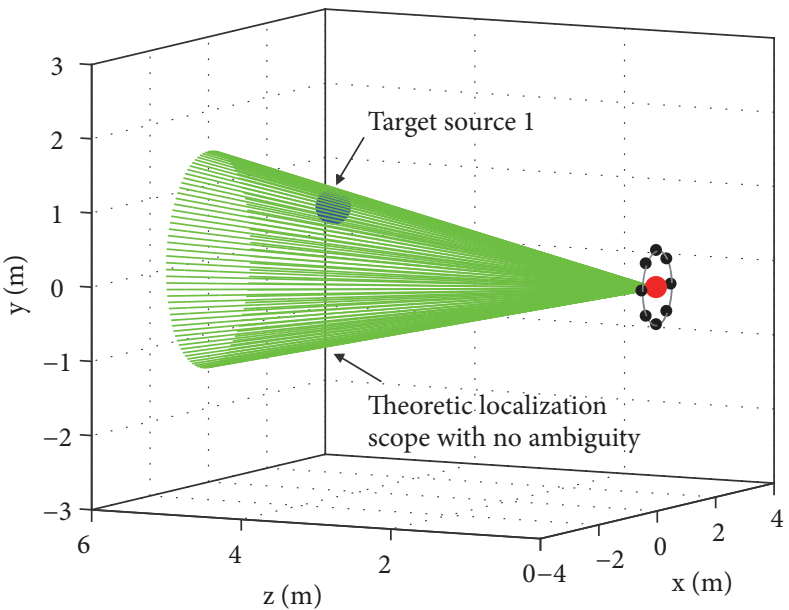

(a)

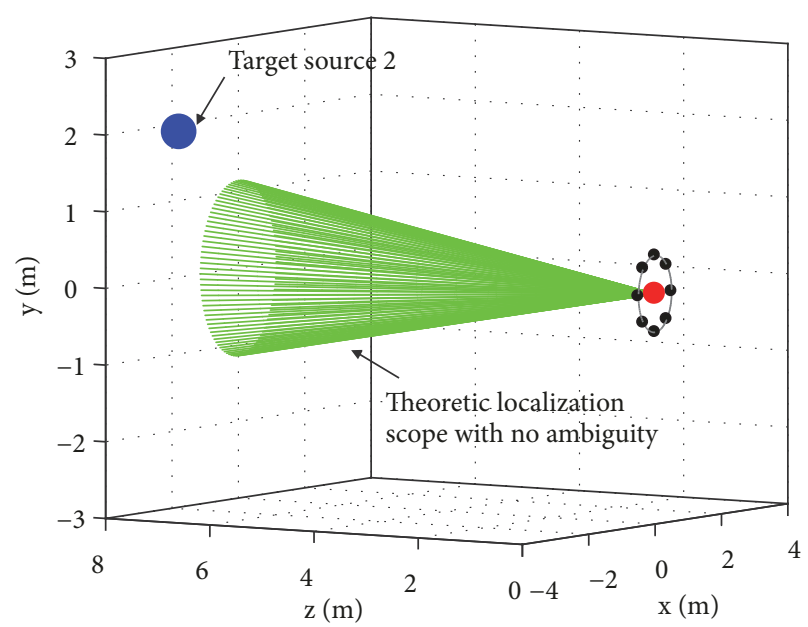

(b)

Figure 5: Source's 3D location. (a) Source $1(f=900 \mathrm{MHz})$. (b) Source $2(f=1.5 \mathrm{GHz})$.

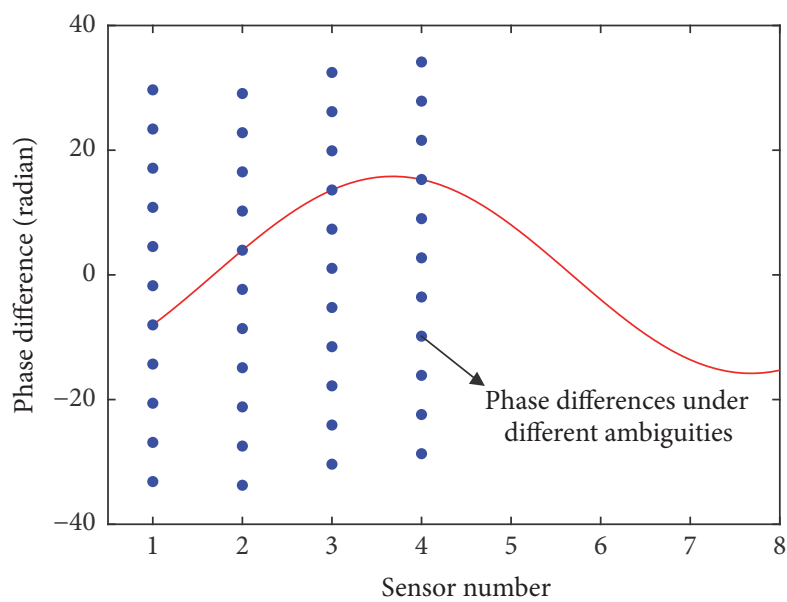

Unambiguous cosine curve

(a)

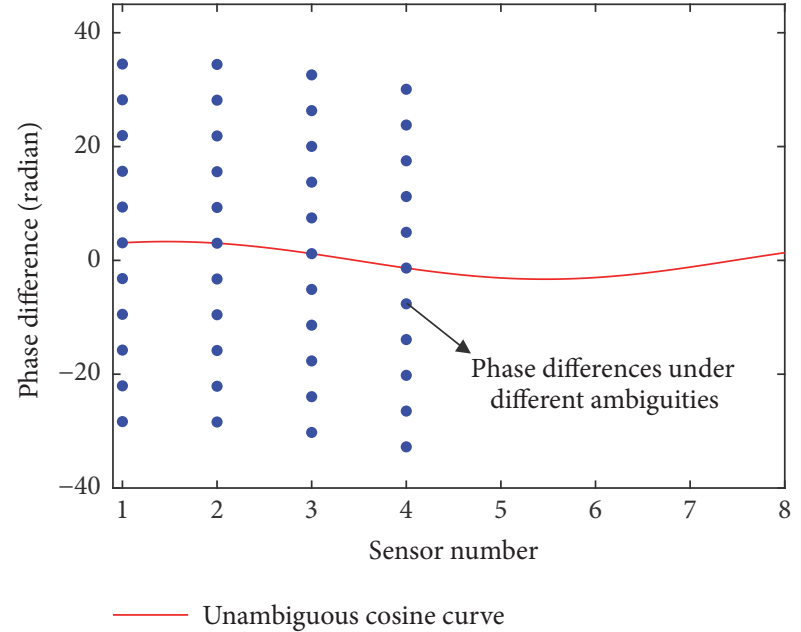

(b)

FIgURE 6: Phase differences under different ambiguities. (a) Source 1. (b) Source 2.

located at the unambiguous zone, while the location of source with $f=1.5 \mathrm{GHz}$ is located outside the unambiguous zone.

After computing the phase differences of CSSs, phase difference matrix could be developed by ambiguity traversing. It can be seen from the Figure 6 that blue dots represent the phase differences under different ambiguities and the red curve represents the target cosine function corresponding to unambiguous phase differences. Note that four phase difference dots are just located at the cosine curve. It should be pointed out that there exist multiple cosine curve lines in the phase difference matrix, but only one normal cosine curve corresponds to unambiguous phase differences.

Thus, we could employ the method of exhaust searching and cosine matching to ascertain unambiguous phase differences. Herein, in order to directly exhibit the rationality of method, we utilize elevation searching to substitute aforementioned amplitude searching and the step size in angle searching is $\Delta \theta=1^{\circ}$. The sum of minimum differences is presented in Figure 7 . It is clear that the minimum of sum just corresponds to the actual source's angles. Therefore, by searching the minimum, source's rough angle estimation with no ambiguity can be obtained.

Figure 8 shows the comparison of source's angle estimation by using our proposed ATCM method and the phasebased algorithm in [12]. For angle estimation of source 1, which is located at the unambiguous position, both the two methods can achieve accurate localization. However, for angle estimation of source 2 , which is located outside the unambiguous zone, the phase-based algorithm is ineffective, while the ATCM method can resolve ambiguity and obtain actual source's angles. 


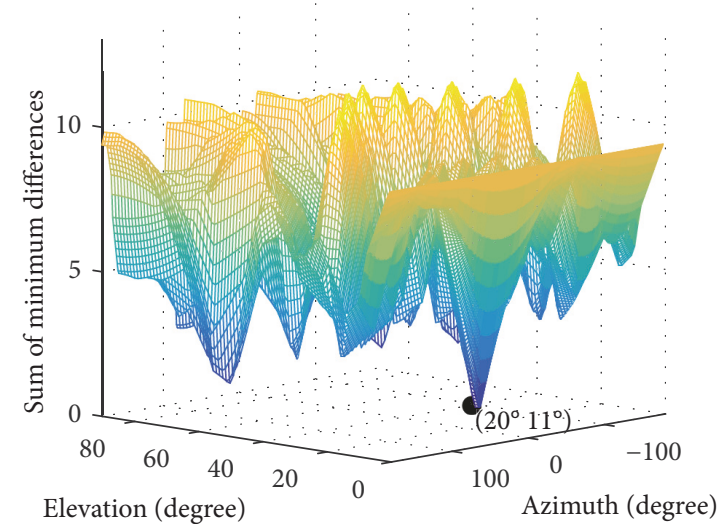

(a)

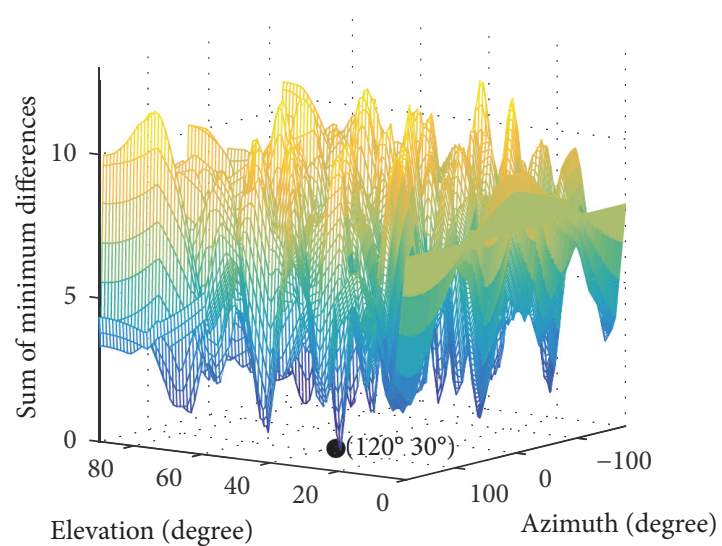

(b)

Figure 7: Sum of minimum difference. (a) Source 1. (b) Source 2.

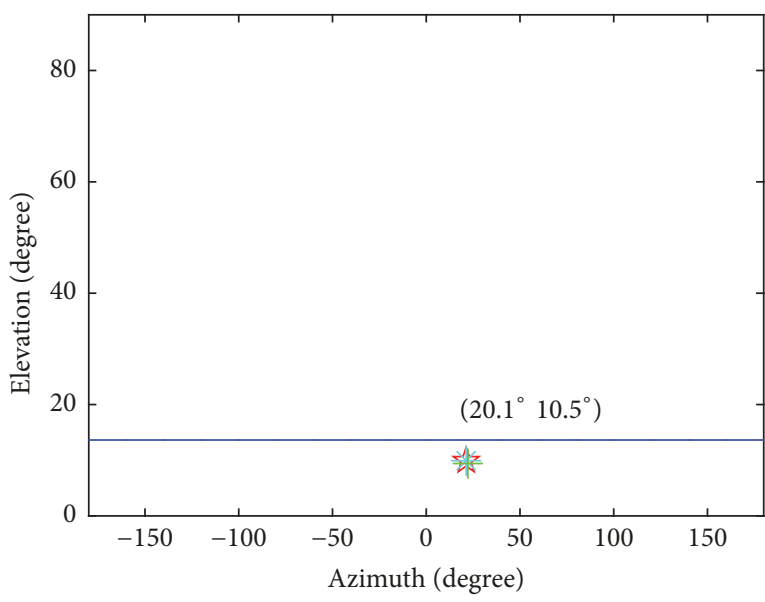

is Actual angle location of source 1

* Angle estimation of the literature [12]

+ Angle estimation of the proposed algorithm

_ Maximum unambiguous elevation

(a)

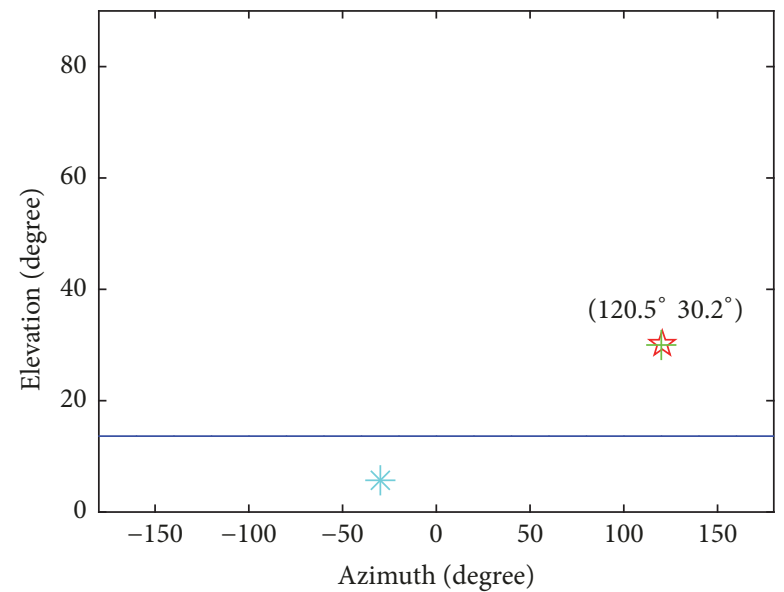

is Actual angle location of source 2

* Angle estimation of the literature [12]

+ Angle estimation of the proposed algorithm

— Maximum unambiguous elevation

(b)

FIgURE 8: Comparison of source's angle estimation. (a) Source 1. (b) Source 2.

5.3. The Performance Analysis of ATCM. In this part, we first define the precise estimation as the difference between parameter estimation and actual value is no more than one degree. The probability of precise estimation $P_{p e}=$ $\left(N_{p e} / N_{m o}\right) \cdot 100 \%$ representing the ratio between the count of precise estimation $N_{p e}$ and Monte Carlo runs $N_{m o}$ is then utilized to present the performance of ATCM. We have pointed out that the estimation precision mainly depends on the step size in search. Therefore, considering three typical source's frequencies ( $f=1 \mathrm{GHz}, 2 \mathrm{GHz}, 3 \mathrm{GHz}$ ), three hundred Monte Carlo runs are performed to obtain the probability of precise source's angle estimation. Assuming that the distribution of source's location is random, the sampling rate is $f_{s}=6 \mathrm{GHz}$, snapshots $N=6000$, the number of sensors is set as $M=8$, the spacing $l=2$, and other experimental parameters are kept unchanged. It can be seen from Figure 9(a) that the probability decreases as the step size increases, and only when the step size is less than 0.2 degree, the probabilities under different source's frequencies are near to a hundred percent. However, the smaller the step size, the higher the computational cost. The elapsed CPU times of the ATCM algorithm by employing the step sizes $\Delta \theta=0.2^{\circ}$ and $\Delta \theta=1^{\circ}$ for a single run are measured as $0.57 \mathrm{~s}$ and $13.61 \mathrm{~s}$, respectively. Meanwhile, we also study the influence of SNR on the ATCM algorithm by employing the step size $\Delta \theta=0.2^{\circ}$. As shown in Figure 9(b), the probability of precise estimation obviously improves as SNR increases, and the amplitude of change is gentle when the SNR is larger 


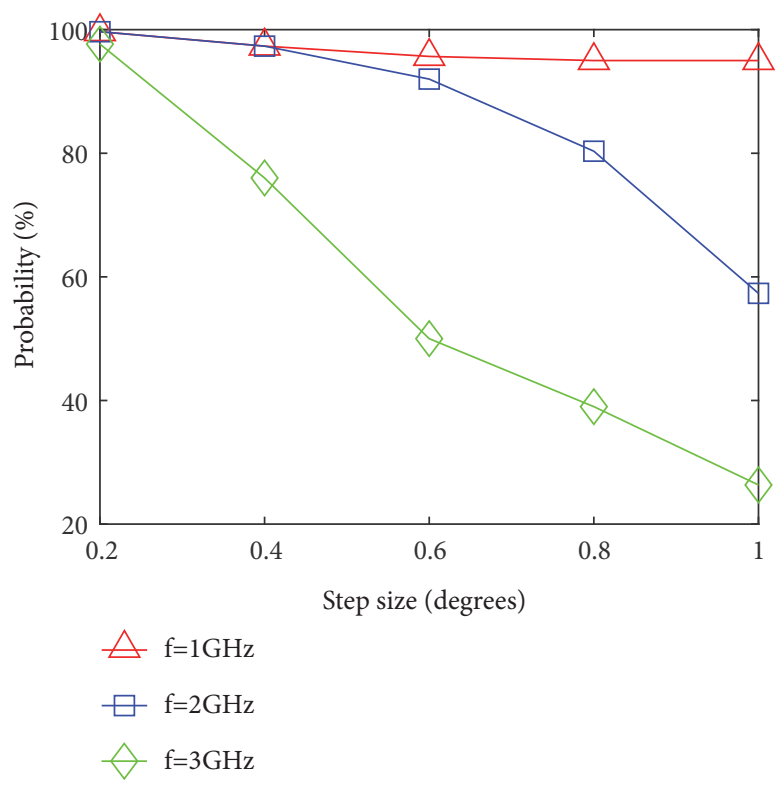

(a)

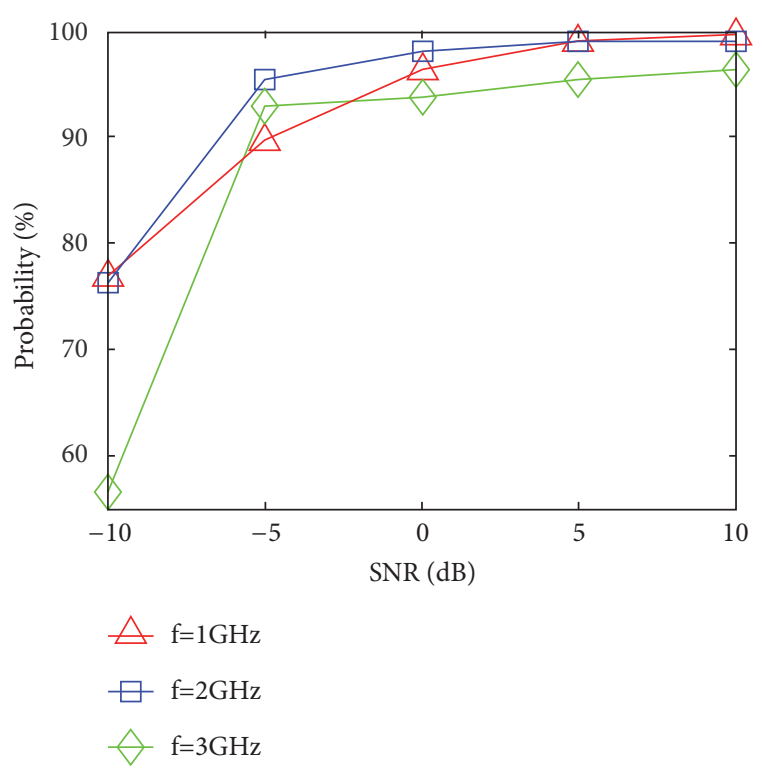

(b)

FIGURE 9: Probability of precise estimation versus step size and SNR. (a) Step size in search (noiseless). (b) SNR (step size = 0.2).

than $0 \mathrm{~dB}$. Moreover, the probability of precise estimation is acceptable under different frequencies of estimated source at high SNR.

As a whole, the proposed ATCM algorithm with small step size in search is more feasible for moving sources. However, for fixed sources, it can always employ relatively large step size to obtain rough source's angles with no ambiguity, which has obvious advantage over small step size in computational cost.

5.4. The Estimation Performance of Source's 3D Localization. The rough source's angle estimation obtained by ATCM can be utilized to resolve ambiguity in phase-based algorithm in [12], and source's range as well as more precise angle estimation can be realized. To demonstrate the effectiveness of this scheme and the satisfactory performance of source's 3D localization, three hundred Monte Carlo runs are performed to obtain histogram distribution of source's parameter estimation. The experimental parameters are identical to Section 5.2. It should be noticed from Figures 10(a), 10(b), and 10 (c) that the histogram distribution of source's $3 \mathrm{D}$ parameter estimation is near to the true value, and the times located at true value are relatively larger than others. The maximum estimation errors of azimuth angle, elevation angle, and range approximate to $0.05^{\circ}, 0.01^{\circ}, 0.8 \mathrm{~m}$, respectively. In Figures $10(\mathrm{~d}), 10(\mathrm{e})$, and $10(\mathrm{f})$, the situation is similar.

As a whole, our proposed method can achieve both ambiguous and unambiguous source's $3 \mathrm{D}$ localization with excellent estimation performance.

\section{Conclusion}

3D source localization of an over-frequency source always suffers from phase ambiguity under UCA with a fixed array diameter. This paper first investigates the correct localization zone using phase-based algorithms and a novel ambiguity resolution method based on the cosine property of ambiguity-free phase differences is then presented, which can achieve multiple incoherent monofrequency as well as frequency-hopping sources' unambiguous 3D parameter estimation. Simulation experiments demonstrate the validity of theoretical analysis on unambiguous estimation zone and the effectiveness of ambiguity resolution algorithm. Research results could provide useful guidance in ambiguity analysis and practical appliance if the phase-based algorithms are to be implemented.

\section{Data Availability}

The data used to support the findings of this study are available from the corresponding author upon request.

\section{Conflicts of Interest}

The authors declare no conflicts of interest.

\section{Authors' Contributions}

The main idea was proposed by Zhen Liu and Xin Chen; Xin Chen and Zhenhua Wei conceived and designed the experiments; Zhen Liu and Tianpeng Liu wrote the paper; Linlin Li and Bo Peng revised the paper.

\section{Acknowledgments}

This work is supported in part by the National Natural Science Foundation of China (no. 61401481) and in part by 


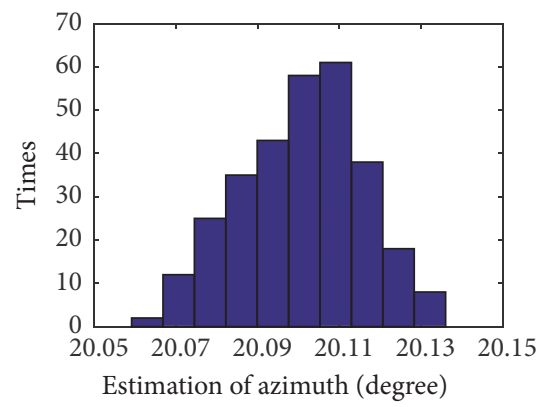

(a)

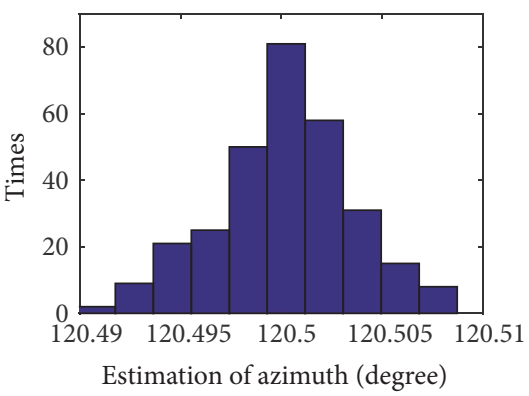

(d)

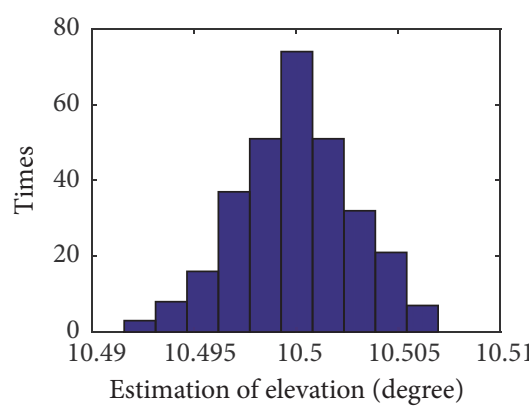

(b)

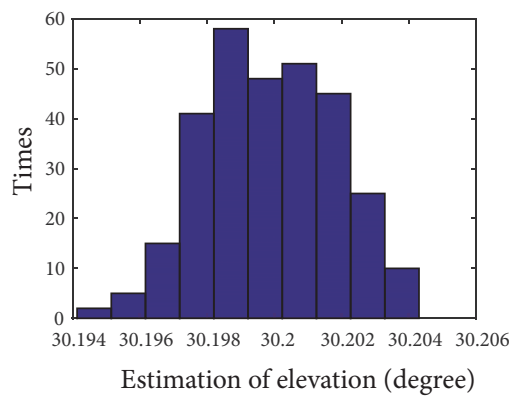

(e)

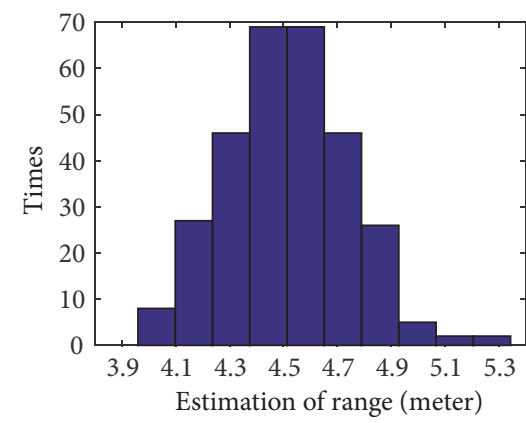

(c)

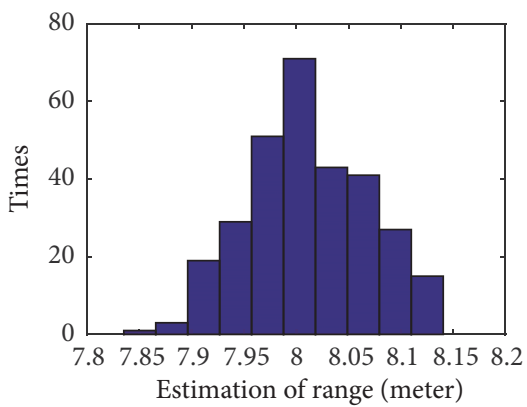

(f)

Figure 10: Histogram distribution of source's 3D parameter estimation. (a) Azimuth angle $(f=900 \mathrm{MHz})$. (b) Elevation angle $(f=900 \mathrm{MHz})$. (c) Range $(f=900 \mathrm{MHz})$. (d) Azimuth angle $(f=1.5 \mathrm{GHz})$. (e) Elevation angle $(f=1.5 \mathrm{GHz})$. (f). Range $(f=1.5 \mathrm{GHz})$.

the Natural Science Foundation of Hunan Province, China (no. 2017JJ3368).

\section{References}

[1] Y. D. Huang and M. Barkat, "Near-field multiple source localization by passive sensor array," IEEE Transactions on Antennas and Propagation, vol. 39, no. 7, pp. 968-975, 1991.

[2] S. Zhou and P. Willett, "Submarine location estimation via a network of detection-only sensors," IEEE Transactions on Signal Processing, vol. 55, no. 6, pp. 3104-3115, 2007.

[3] M. Morinaga, H. Shinoda, and H. Kondoh, "DOA estimation of coherent waves for $77 \mathrm{GHz}$ automotive radar with three receiving antennas," in Proceedings of the 6th European Radar Conference, pp. 145-148, Rome, Italy, 2009.

[4] R. O. Schmidt, "Multiple emitter location and signal parameter estimation," IEEE Transactions on Antennas and Propagation, vol. 34, no. 3, pp. 276-280, 1986.

[5] B.-S. Kwon, T.-J. Jung, C.-H. Shin, and K.-K. Lee, "Decoupled 3D near-field source localization with UCA via centrosymmetric subarrays," IEICE Transactions on Communications, vol. E94-B, no. 11, pp. 3143-3146, 2011.

[6] N. Wu, Z. Qu, W. Si, and S. Jiao, "DOA and polarization estimation using an electromagnetic vector sensor uniform circular array based on the ESPRIT algorithm," Sensors, vol. 16, no. 12, 2016.

[7] Y. Wu and H. C. So, "Simple and accurate two-dimensional angle estimation for a single source with uniform circular array," IEEE Antennas and Wireless Propagation Letters, vol. 7, pp. 7880, 2008.
[8] Y. Wu, H. Wang, Y. Zhang, and Y. Wang, "Multiple nearfield source localisation with uniform circular array," IEEE Electronics Letters, vol. 49, no. 24, pp. 1509-1510, 2013.

[9] D. Starer and A. Nehorai, "Passive localization on near-field sources by path following," IEEE Transactions on Signal Processing, vol. 42, no. 3, pp. 677-680, 1994.

[10] J. H. Lee, D. H. Park, G. T. Park, and K. K. Lee, "Algebraic path-following algorithm for localising 3-D near-field sources in uniform circular array," IEEE Electronics Letters, vol. 39, no. 17, pp. 1283-1285, 2003.

[11] E.-H. Bae and K.-K. Lee, "Closed-form 3-D localization for single source in uniform circular array with a center sensor," IEICE Transactions on Communications, vol. E92-B, no. 3, pp. 1053-1056, 2009.

[12] T.-J. Jung and K. Lee, "Closed-form algorithm for 3-D singlesource localization with uniform circular array," IEEE Antennas and Wireless Propagation Letters, vol. 13, pp. 1096-1099, 2014.

[13] Y. Wu, H. Wang, L. Huang, and H. C. So, "Fast algorithm for three-dimensional single near-field source localization with uniform circular array," in Proceedings of the 6th International Conference on Radar, RADAR 2011, pp. 350-352, China, October 2011.

[14] L. Zuo, J. Pan, and Z. Shen, "Analytical Algorithm for 3-D Localization of a Single Source with Uniform Circular Array," IEEE Antennas and Wireless Propagation Letters, vol. 17, no. 2, pp. 323-326, 2018.

[15] C. M. Tan, S. E. Foo, M. A. Beach, and A. R. Nix, "Ambiguity in MUSIC and ESPRIT for direction of arrival estimation," IEEE Electronics Letters, vol. 38, no. 24, pp. 1598-1600, 2002.

[16] J. Xin, G. Liao, Z. Yang, and H. Shen, "Ambiguity resolution for passive 2-D source localization with a uniform circular array," Sensors, vol. 18, no. 8, 2018. 
[17] X. Chen, Z. Liu, and X. Wei, "Unambiguous parameter estimation of multiple near-field sources via rotating uniform circular array," IEEE Antennas and Wireless Propagation Letters, vol. 16, pp. 872-875, 2017.

[18] X. Chen, Z. Liu, and X. Z. Wei, "Ambiguity resolution for phasebased 3-d source localization under fixed uniform circular array," Sensors, vol. 17, no. 5, Article ID 1086, 2017.

[19] X. Chen, S. H. Wang, Z. Liu, and X. Z. Wei, "Ambiguity resolving based on cosine property of phase differences for 3-D source localization with uniform circular array," in Proceedings of the 2017 International Conference On Digital Image Processing (ICDIP, Hongkong), China, May 2017. 


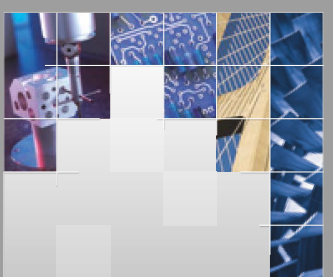

\section{Enfincering}
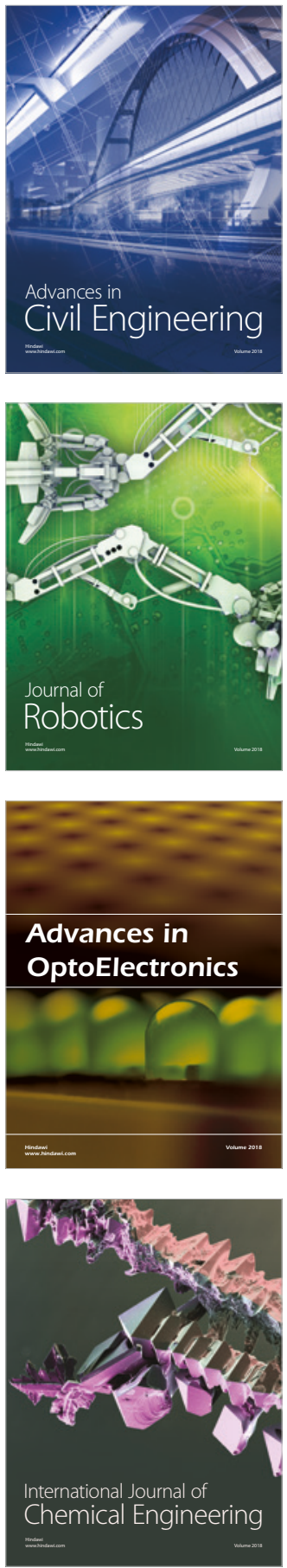

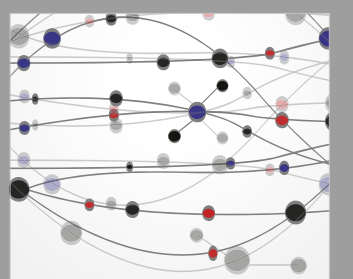

\section{Rotating \\ Machinery}

The Scientific World Journal

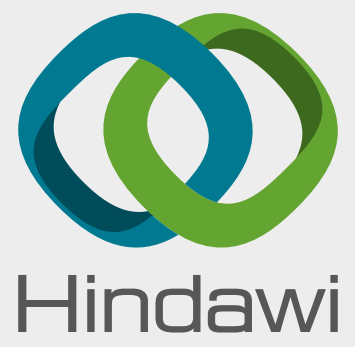

Submit your manuscripts at

www.hindawi.com
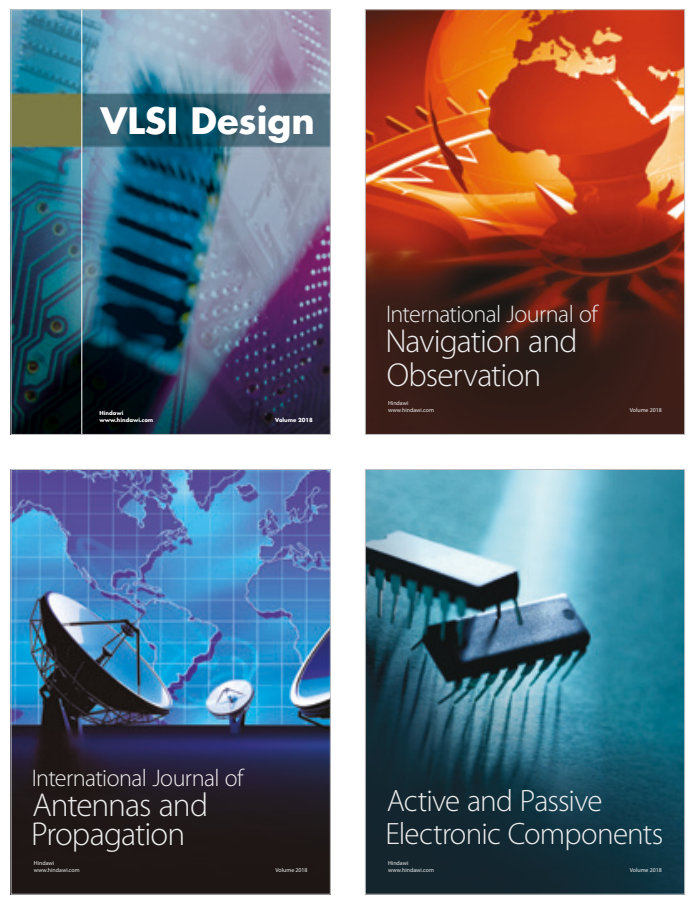
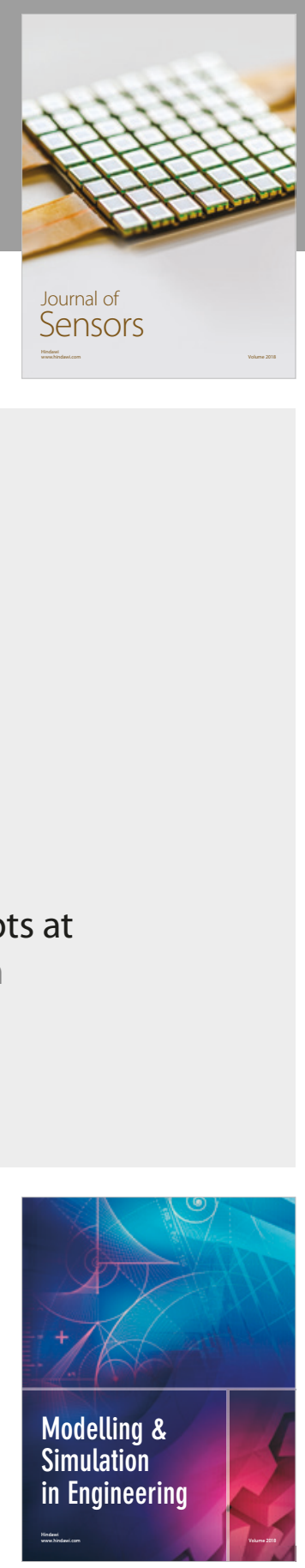

\section{Advances \\ Multimedia}
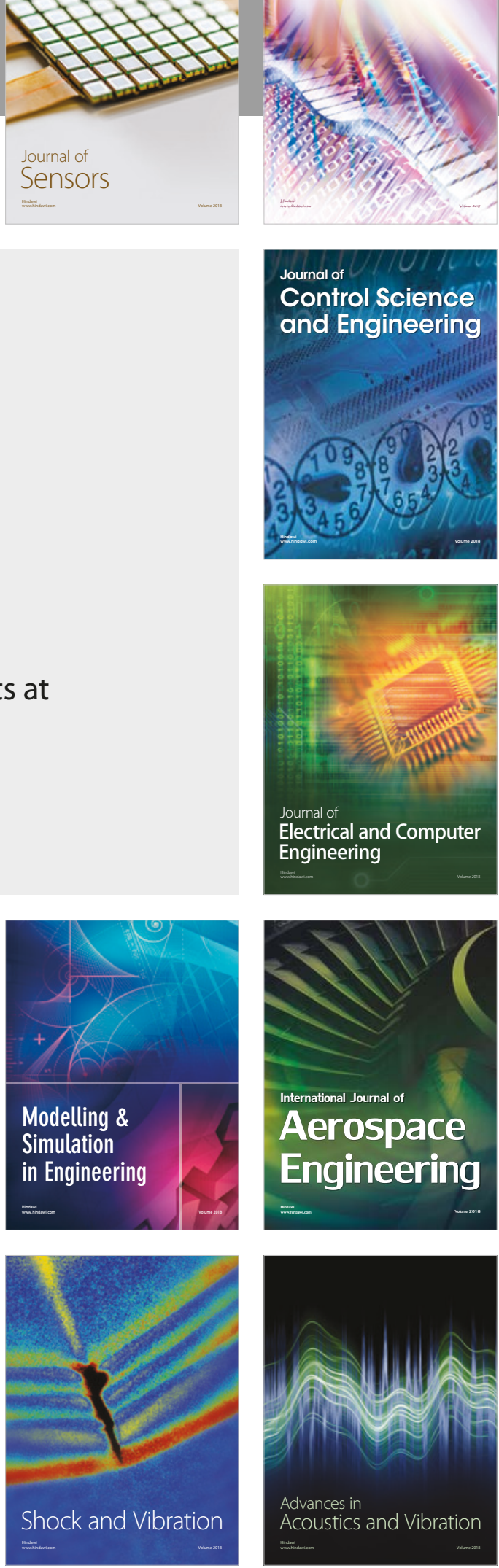